\title{
Rousseau und die Idee einer ,pathologischen' Gesellschaft
}

\author{
Frederick Neuhouser
}

\section{Rousseau and the Idea of a 'Sick' Society}

Abstract: Using resources from the Second Discourse and the Discourse on Political Economy, this paper reconstructs various conceptions of social pathology implicit in Rousseau's work. It argues that good sense can be made of the idea of a social pathology without relying on unduly medical or organic conceptions of society. Special attention is devoted to explaining Rousseau's picture of a "feverish" society, as grounded in "inflamed" forms of amour-propre.

Keywords: Social Pathology, Rousseau, Social Philosophy

Schlagwörter: Soziale Pathologie, Rousseau, Sozialphilosophie

\section{Einleitung}

Eine bemerkenswerte Eigenschaft der verschiedenen Sozialphilosophien des 18., 19., und 20. Jahrhunderts ist die zentrale Rolle, die normative Begriffe der Natur - und nicht nur der menschlichen Natur - in ihren Auffassungen von einer ,guten“ Gesellschaft gespielt haben. ${ }^{1}$ Es ist allgemein bekannt, dass ,Natur ${ }^{6}$ eine wichtige, wenn auch eine verwirrende Rolle in dem sozialpolitischen Denken Rousseaus spielt. An anderer Stelle habe ich behauptet, Rousseaus Berufung auf die Natur ließe sich so interpretieren, dass sich der normative Inhalt des Begriffs auf verhältnismäßig unumstrittene Voraussetzungen beschränkt, die besagen, was Menschen auf Grund ihrer biologischen Natur geneigt sind, zu suchen und als unverzichtbare Bestandteile eines guten Lebens zu betrachten (Neuhouser 2009). In diesem Aufsatz möchte ich das Verhältnis zwischen Natur und ethischer Bewertung aus einer anderen Perspektive betrachten, indem ich die normative Rolle untersuche, die ein aus der Biologie stammender Begriff - der der Krankheit oder der Pathologie - in seiner Sozialphilosophie spielt. Eine große Anzahl von späteren Denkern - man denke zum Beispiel an Hegel, Nietzsche, oder Durkheim - stützen sich bei der ethischen Beurteilung von Gesellschaften auf die Idee der Pathologie. Aber Krankheitsmetaphern sind auch bei Rousseau zu finden. Sein Diskurs über den Ursprung der Ungleichbeit (der „Zweite Diskurs“) kann als ein erweitertes Argument dafür gelesen werden, dass die moderne Gesellschaft , $\mathrm{krank}^{6}$ ist. Dieser Eindruck wird in der ersten Fassung des Gesellschaftsvertrags bestätigt, wenn Rousseau verschiedene gesellschaftliche Krankheiten beschreibt und dann seine Absicht verkündet, „aus dem Übel selbst das Gegenmittel zu gewinnen, das es heilen wird“ (Rousseau 1995, S. 301; Rousseau 1964, S. 288). In einer neueren

1 Für Hilfe beim Verfassen dieses Aufsatzes auf Deutsch bin ich Christian Heilbronn sehr dankbar. 
Arbeit habe ich behauptet, Rousseau finde sowohl die Ursache als auch deren eventuelles Heilmittel in der Leidenschaft der amour-propre, das heißt, in dem menschlichen Streben danach, eine Stellung in den Augen anderer zu gewinnen (Neuhouser 2012). Es ist nicht Ziel dieses Aufsatzes zu erklären, warum die amour-propre der gefährlichste und zugleich der vielversprechendste Bestandteil der menschlichen Natur ist. ${ }^{2}$ Stattdessen soll er einigen Gedanken über den Begriff der sozialen Pathologie aufstellen, der Rousseaus Kritik der modernen Gesellschaft zugrunde liegt. Die leitende Fragen dieses Aufsatzes sind: Inwiefern ist es sinnvoll zu behaupten, eine Gesellschaft - die offensichtlich kein biologischer Organismus ist - sei ,krank'? Und wie könnte eine Diagnose von sozialer Pathologie uns sozialkritische Mittel liefern, die die Grenzen des bekannteren Begriffs der Ungerechtigkeit überschreiten? Anders formuliert: Welche kritikbedürftigen gesellschaftlichen Phänomene geraten nicht in unser Blickfeld, wenn wir uns nur auf Begriffe beschränken, die aus der liberalen politischen Philosophie stammen?

\section{Der Begriff der sozialen Pathologie: erste Annäherung}

Die Idee, Gesellschaften können krank sein, ist mindestens so alt wie Platons Staat, der seine Behandlung der Gerechtigkeit mit einem Vergleich zwischen einer ,gesunden' und einer ,fieberhaften' Polis beginnt (Plato 2004, 369-374e). Für dieses Thema ist Rousseau besonders interessant, weil er sich weniger auf biologische Vergleiche stützt ${ }^{3}$ als Platon und Philosophen des 19. Jahrhunderts es taten. Da Rousseau in seiner Sozialphilosophie biologische Vergleiche meistens vermeidet, ist es weniger klar, welchen Sinn es haben könnte, eine Gesellschaft als ,krank' zu bezeichnen.

Es gibt eine relativ einfache Auffassung der sozialen Pathologie, die die menschliche Gesellschaft nicht als Organismus versteht, die aber eine Verbindung zwischen sozialer Pathologie und Biologie aufstellt, indem sie die Befriedigung der biologischen Bedürfnisse der Menschen als eine Hauptfunktion der Gesellschaft ansieht. Nach dieser Auffassung besteht die soziale Pathologie in der Unfähigkeit einer Gesellschaft, die materiellen Bedingungen des menschlichen Lebens zu reproduzieren. Es gibt verschiedene Phänomene, die in diesem Sinn als soziale Pathologien gelten könnten - zum Beispiel, Geburtsraten, die zu niedrig sind, als dass sich die Gesellschaft über Generationen reproduzieren könnte, oder ökonomische Praktiken, die die Umwelt und dadurch die Bedingungen des menschlichen Überlebens zerstören. Solche Phänomene gelten als soziale, statt biologische, Pathologien, wenn sie soziale statt biologische Ursachen haben - wenn, zum Beispiel, unser Kollisionskurs mit der Natur darauf gegründet wäre, was Marx die ,gesellschaftliche Form' der Produktion nannte. Rousseau erkennt auf implizite Weise diese Art von sozialen Pathologien, wenn er im Gesellschaftsvertrag die

2 ,Die menschliche Natur` bezieht sich hier nicht auf die ,ursprüngliche ${ }^{`}$ Natur des Menschen (die Eigenschaften, die isolierte Individuen im ursprünglichen Naturzustand, wie er im Zweiten Diskurs geschildert wird, haben). Gemeint ist die menschliche Natur im breiteren Sinne - die grundlegenden Fähigkeiten und Leidenschaften, die alle vergesellschaftlichten Menschen besitzen).

3 Zwei Ausnahmen sind an folgenden Stellen zu finden: (Rousseau 1964, S. 244) and (Rousseau 1964, S. 424). 
Gesundheit einer Gesellschaft in einer sich vermehrenden Anzahl ihrer Einwohner sieht (Rousseau 1964, S. 420). Aber auch wenn die unser biologisches Überleben bedrohenden sozialen Pathologien für uns heute die dringendsten Fälle darstellen, sind sie weit davon, die philosophisch interessantesten Fälle zu sein. Trotzdem gibt es vier Merkmale dieser einfachsten Fälle, die auch in den komplexeren Fällen zu finden sind.

Erstens beinhaltet die Behauptung, eine Gesellschaft sei krank, mehr als die einfache Feststellung, sie sei ungerecht. Das heißt nicht, dass der Begriff der Ungerechtigkeit allen Diagnosen einer sozialen Pathologie irrelevant ist, sondern dass, auch wenn er relevant ist, die Bezeichnung einer Pathologie als ,Ungerechtigkeit‘ immer zu wenig über das Phänomen aussagt. Es ist plausibel, zum Beispiel, die Erderwärmung als ein Fall von Ungerechtigkeit zu betrachten (eine Ungerechtigkeit an zukünftige Generationen vielleicht, oder eine Ungerechtigkeit auf Seiten deren, die davon profitieren). Es wäre aber ein Fehler, dies als eine erschöpfende Beschreibung des Phänomens zu betrachten. Das ist sogar noch einleuchtender in dem Fall von zu niedrigen Geburtsraten, wo es schwieriger ist, das Problem als Ungerechtigkeit an zukünftige Generationen anzusehen. In diesem Beispiel lässt sich der Eindruck kaum vermeiden, dass irgendwas ernsthaft schief gegangen ist - dass irgendeine Krankheit vorhanden ist - wenn soziale Praktiken Folgen haben, die grundlegenden menschlichen Zwecken zuwiderlaufen (in diesem Fall Zwecken, die aus unserer biologischen Natur stammen). Bei der Erderwärmung ist der Eindruck, eine Krankheit sei vorhanden, noch schwieriger zu vermeiden, wenn Menschen, nachdem sie der Gefahr schon einmal bewusst geworden sind, dieselben Praktiken fortsetzen, die die Bedingungen ihres Überlebens unterhöhlen.

Aber was steckt hinter dem Eindruck, dass man in solchen Fällen von Pathologien sprechen kann? Eine Antwort liegt in der Idee einer Praktik, die den eigenen Zwecken derer, die an dieser Praktik teilnehmen, systematisch zuwiderläuft. Wenn das so ist, dann hängt die Idee der sozialen Pathologie von der Vorstellung ab, man könne sozialen Praktiken Zwecke, auch kollektive Zwecke, zuschreiben und eine soziale Pathologie sei dann vorhanden, wenn das normale Funktionieren jener Praktiken entweder ihren eigenen Zwecken oder anderen grundlegenden Zwecken der Gesellschaft auf eine nicht zufällige Weise zuwiderläuft. Ich lasse hier zur Seite die philosophischen Fragen, die sich ergeben, wenn man sozialen Praktiken Zwecke zuschreibt, um stattdessen auf den Grund zu reflektieren, weshalb Theorien, die die Idee der sozialen Pathologie verwenden, dazu tendieren, den Rahmen der liberalen Auffassungen der Gerechtigkeit zu sprengen. Der Grund dafür liegt darin, dass der Mangel, der eine soziale Pathologie auszeichnet, ein Mangel an der Verwirklichung des Guten statt des Rechten ist. Obwohl das nicht auf alle sozialen Pathologien zutrifft, ist es meistens der Fall, dass die Beschreibung einer Gesellschaft als ,krank' davon abhängt, dass man ihr Zwecke zuschreibt, die mit Auffassungen des Guten oder des menschlichen Gedeihens verbunden sind, die ,dicker' sind als die, die liberale Gerechtigkeitstheorien zulassen. Das ist allerdings als kein Mangel der Theorien, die sich auf einen Begriff der sozialen Pathologie stützen, sondern eine konsequente Folge davon, dass ihr Gegenstand soziale, nicht bloß politische Realität ist. Eine Theorie, die die Idee der sozialen Pathologie ernst nimmt, beruht auf einer Auffassung des Sozialen, nach 
dem es unmöglich ist, Gesellschaften zu verstehen oder zu bewerten, ohne sozialen Praktiken Zwecke zuzuschreiben, die in einem weiten Sinn ethisch sind.

Ein zweites Merkmal auch der einfachsten Fälle von sozialer Pathologie ist, dass sie nicht in abgesonderten Ereignissen sondern in andauernden Praktiken bestehen, die eine identifizierbare Logik oder Dynamik besitzen. Das ist genau der Punkt, der hinter der von Max Weber getroffenen Unterscheidung zwischen vereinzelten auf Profit gerichteten Tätigkeiten und dem, was er ,kapitalistischer Betrieb' nennt, steckt. Das auszeichnende Merkmal des letzteren liegt darin, dass es in einer kontinuierlich wiederholten Reihe von Tätigkeiten besteht, die von einem spezifischen Ziel vereinigt wird (Maximierung des Profits), und die eine gewisse Struktur darstellt (ökonomische Effizienz, die durch marktorientierte Berechnungen der Rentierbarkeit bestimmt wird). Solche Praktiken haben nicht nur Zwecke sondern auch eine charakteristische Dynamik, die deren Teilnehmern auch unbewusst bleiben kann. Die Erderwärmung, zum Beispiel, lässt sich nicht als vereinzelte Handlungen von achtlosen Individuen verstehen; sie ist vielmehr das Ergebnis einer sozialen Praktik, die ihre eigene, durch das Ziel der Kapitalakkumulation bestimmte Logik besitzt, welche nicht ohne die reelle Umgestaltung von Institutionen zu verändern ist. Wenn man die physiologische Pathologie als eine Störung eines andauernden funktionalen Prozesses eines Organismus versteht, die jene Funktion unterhöhlt oder die eine Gefahr für die Lebenskraft des Organismus darstellt, dann lassen sich auch soziale Praktiken, die eine ähnliche Dynamik besitzen, als Pathologien begreifen.

Drittens muss eine soziale Pathologie gesellschaftliche, nicht bloß natürliche Ursachen haben. Wenn die Erderwärmung ein rein natürlicher Prozess wäre wenn etwa die Ozonschicht zerstörenden Gase natürliche Abfallprodukte von normalen biologischen Prozessen wären - könnte man sie als ein sich selbst zuwiderlaufendes Phänomen beschreiben, aber sie wäre keine soziale Pathologie, weil sie ja kein soziales Phänomen wäre. Ein Teil dessen, was ein soziales Phänomen ausmacht, liegt darin, dass es sich aus Bedingungen ergibt, die von uns (statt durch die Natur) gemacht worden sind, und die deshalb im Prinzip von uns verändert werden können. Soziale Praktiken sind Prozesse, an denen wir aktiv teilnehmen, und für die wir deshalb verantwortlich sind. Oder, wie Rousseau es ausdrückt, hängen soziale Praktiken von der ,Einwilligung' ihrer Teilnehmer ab (Rousseau 1993, S. 67; Rousseau 1964, S. 131). ${ }^{4}$ Aus diesem Grund beinhaltet eine Diagnose der sozialen Pathologie immer ein Moment der Kritik, das bei der Diagnostizierung von biologischen Pathologien fehlt. Weil sie von der menschlichen Freiheit abhängen, gehören soziale Phänomene, im Gegensatz zu rein natürlichen Prozessen, dem Reich des Normativen an. Und dies erklärt, warum eine Diagnose von sozialer Pathologie auch in einem zweiten Sinn ethisch ist: nicht nur beruht sie auf Auffassungen dessen, was für Menschen gut ist, sondern sie betrachtet auch ihre Adressaten als verantwortliche Akteure.

Beim vierten Punkt geht es um eine weitere Hinsicht, in der eine Diagnose von sozialer Pathologie ethisch ist: Auch wenn die Zwecke, nach welchen wir eine

4 Rousseau redet hier von sozialen, oder , moralischen', Ungleichheiten, aber der Punkt gilt auch für soziale Praktiken. 
soziale Praktik bewerten, uns von der Biologie gegeben sind (wie bei der Erderwärmung oder bei niedrigen Geburtsraten), betrachtet der Sozialkritiker den relativen Wert jener natürlichen Zwecke nicht als von der Natur festgelegt. Die Auffassung der Erderwärmung als einer Pathologie beruht, zum Beispiel, auf einer negativen Bewertung der Zwecke, denen die produktiven Praktiken dienen, die die schädlichen Gase als Nebenprodukte abgeben. Wenn dieselben produktiven Praktiken unternommen würden, um allen Menschen einen würdigen Lebensstandard zu ermöglichen, würde uns die Tatsache, dass wir dabei schädliche Gase produzieren, viel weniger als eine Krankheit erscheinen, als wenn die lebensbedrohende Produktion bloß der Bereicherung einiger Individuen diente, die wiederum die Schaffung und Befriedigung von immer größeren Begierden nach benzinsaufenden Autos, billigen Urlaubsflügen, und Massenvernichtungswaffen verlangte. Wenn so ein Szenario als eine Krankheit betrachtet wird, setzt man implizit den Wert des menschlichen Lebens in Verhältnis zu dem Wert der anderen Zwecke, denen die fraglichen produktiven Praktiken dienen. Das bedeutet, dass soziale Kritik auch in Fällen, wo die Diagnose der sozialen Pathologie nur von Werten abhängt, die „von der Natur" kommen, durch und durch eine ethische ist.

\section{Das Beispiel Roms: fünf weitere Aspekte von sozialen Pathologien}

In diesem Abschnitt geht es um komplexere Fälle von sozialen Pathologien, bei denen die Zwecke, die als Maßstäbe der Kritik verwendet werden, nicht aus unseren Interessen als biologische Wesen stammen. Es gibt viele Möglichkeiten, soziale Pathologien auf diese Weise zu verstehen, aber hier sollen nur Ressourcen, die in Rousseaus Sozialphilosophie zu finden sind, berücksichtigt werden. Eine reichhaltige aber eher unbekannte Textstelle aus dem Diskurs über die Politische Ökonomie liefert dem Leser deutliche Beispiele von sozialen Pathologien:

„Es kann eine Zeit kommen, wo sich die Bürger nicht mehr der gemeinsamen Sache verpflichtet fühlen und es ablehnen, das Vaterland zu verteidigen, und wo die Magistraten lieber Söldner kommandieren wollen als freie Menschen, und wäre es nur, um die ersten [...] dazu zu verwenden, um die zweiten zu unterwerfen. Das geschah in Rom [...] unter den Kaisern. Denn alle Siege der ersten Römer [...] waren von tapferen Bürgern errungen worden, die notfalls ihr Blut für das Vaterland hergaben, es aber nie verkauften. Marius war der erste, der [...] die Legionen entehrte, indem er [...] Söldner [in sie] aufnahm. Nachdem sie die Feinde der Völker geworden waren, die sie glücklich zu machen verpflichtet waren, stellten die Tyrannen Berufstruppen auf, die scheinbar die Fremden zurückhalten, aber in Wirklichkeit die Einwohner unterdrücken sollten. Um diese Truppen aufzustellen, musste man sie den Ackerbauern entreißen. Das Fehlen dieser Leute verminderte die Qualität der Nahrungsmittel. Deren Einfuhr führte zu Steuern, die die Preise erhöhten. Diese ersten Unordnungen ließen die Völker murren. Um sie zum Schwiegen zu bringen, musste man die Truppen verstärken und folglich das Elend. In dem Maß, wie die Hoffnungslosigkeit größer wurde, musste man sie wieder vergrößern, um diesen Auswirkungen vorzubeugen. Im übrigen waren diese Söldner, deren Wert man vom Preis ablesen konnte, zu dem sie sich verkauften, stolz auf ihre Erniedrigung und verachteten die Gesetze [...] und ihre Brüder, deren Brot sie aßen. Sie glaubten, größere Ehre zu haben, wenn sie die Gefolgsleute des Caesars waren, als die Verteidiger Roms. [...] [Sie] hielten den 
gezückten Dolch über ihre Mitbürger und waren bereit, auf das erste Zeichen hin, alles zu ermorden. Der Beweiß wäre nicht schwer, dass das einer der Hauptgründe für den Untergang des römischen Reiches war“ (Rousseau 1977, S. 45-46; Rousseau 1964, S. 268-269).

Diese Geschichte von Rom - mit nur einigen Veränderungen könnte sie als die Geschichte der USA nach dem 11. September 2001 gelten - beschreibt die verheerenden Folgen, die sich ergeben, wenn sich eine Gesellschaft auf „Homeland Security“-Maßnahmen verlässt, um eine Lösung für grundlegende wirtschaftliche, ethische, und politische Probleme zu finden. Obwohl es einige kausalen Lücken in dieser Darstellung der Übel Roms gibt, ist es nicht schwierig, soziale Pathologien darin zu erkennen, und im folgenden soll auf fünf Hinsichten hingewiesen werden, in welchen die hier beschriebene Gesellschaft als ,krank' bezeichnet werden könnte.

Die erste davon liegt in einem Mangel an der Art von ziviler Einheit, die einer ,gesunden' Gesellschaft zukommt. Dieser Mangel an ziviler Einheit - ein Fehlen der angemessenen solidarischen Bände unter Gesellschaftsmitgliedern - sieht man am deutlichsten in der Unfähigkeit der Bürger, eine Konvergenz zwischen ihren Interessen und den Interessen ihrer Mitbürger zu erkennen. Wie Rousseau es hier beschreibt, besteht das Problem in einer bestimmten Weise, wie Bürger sich selbst und ihr Verhältnis zur Gesellschaft wahrnehmen. Für ihn gelten diese Wahrnehmungen der Bürger allein als eine soziale Pathologie, aber diese Abwesenheit von ziviler Einheit ist weit davon, bloß ein Fall von falschem Bewusstsein zu sein. Denn die Wahrnehmungen der Bürger spiegeln eine Wirklichkeit wider, insofern ihre Gesellschaft tatsächlich in feste Klassen - Bürger, Magistraten, und bezahlte Gefolgsmänner - aufgeteilt ist, deren Mitglieder in dem Maße gegensätzliche Interessen verfolgen, dass eine Überstimmung darüber, was das gemeinsame Wohl sei, nicht mehr möglich ist. (Die Magistrate sind an die Beherrschung der Bürger interessiert, während die Bürger daran interessiert sind, genug zu essen zu haben und unbeherrscht zu sein.) Das heißt, dass es in Rom, wie es hier beschrieben wird, sowohl die objektiven als auch die subjektiven Bedingungen fehlen, die einen allgemeinen Willen möglich machen.

Es ist wichtig, zu verstehen, warum Rousseau der zivilen Einheit so eine große Bedeutung zuschreibt. Das Problem ist nicht nur, dass Bürger unter der ungerechten Herrschaft ihrer Gebieter leiden; genau so wichtig ist die Tatsache, dass es einen Riss im gesellschaftlichen Gefüge gibt, der ein gesundes gemeinsames Leben unmöglich macht, wobei das Wort ,gesund' uns nicht zur Vorstellung verleiten sollte, die Wichtigkeit der zivilen Einheit liege außerhalb des Reichs des Normativen. Rousseaus Ansicht nach liegt die Wichtigkeit der zivilen Einheit darin, dass sie die Bildung eines allgemeinen Willens möglich macht, ohne welchen die Bürger nicht „moralisch frei“ sein können (Rousseau 1964, S. 365) - das heißt, eine Art von Freiheit nicht genießen können, deren Fehlen man auch Entfremdung nennen könnte. Für Rousseau gibt es einen wichtigen Unterschied zwischen Beherrschung durch andere Individuen (oder Gruppen) und der politischen Unfreiheit, die darin besteht, dass man Gesetzen unterworfen ist oder an Institutionen teilnimmt, die man nicht als Ausdruck seines eigenen Willens ansehen kann. Im Fall von Rom 
hängt die Unfähigkeit der Bürger, die Gesetze als Produkte ihrer Willen anzusehen, davon ab, dass jene Gesetze ihre eigene Beherrschung hervorbringen. Aber es könnte auch andere Fälle geben, bei denen die Abwesenheit von ziviler Einheit nicht an Ungerechtigkeit läge, und worauf es hier ankommt, ist, dass auch in jenen Fällen das Fehlen von ziviler Einheit ein Übel wäre. Man denke an Fälle, in denen die Gesetze einer Gesellschaft vollkommen gerecht wären, in denen sich aber Individuen nicht mit ihnen identifizierten, weil ihnen die Werte oder die Selbstverständnisse fehlten, die dafür notwendig wären. oder an Fälle, wo ein wirkliches Auseinanderlaufen von besonderen Interessen die Bildung eines allgemeinen Willens unmöglich machte, ohne dass dieses Auseinanderlaufen ein Fall von Ungerechtigkeit darstellte.

Rousseaus Beispiel enthält ein zweites Merkmal, das bei Theorien der sozialen Pathologie häufig auftaucht. Es liegt in der Wichtigkeit, die er der Anstellung von Söldnern zuschreibt - das heißt, in der unangemessenen Einführung von Geld in soziale Beziehungen. Man sollte Rousseau nicht die unplausible Ansicht zuschreiben, die Einmischung von Geld in soziale Beziehungen sei immer zu kritisieren. Plausibler ist die Behauptung, bestimmte Arten von sozialen Beziehungen würden zerstört, sobald Geld zu ihrem leitenden Prinzip wird. Eine von Rousseaus Klagen betrifft die Leichtigkeit, mit der Individuen von den Machthabern manipuliert und im Dienst gemeinschaftsfeindlicher Zwecke benutzt werden können, wenn sie ihre Bürgerpflichten nur deshalb erfüllen, weil sie dafür bezahlt werden. (Söldner lassen sich als Mittel der Beherrschung statt der Förderung des Gemeinwohls benutzen.) Ein verwandter Punkt ist, dass Bürger, die sich kaufen lassen, dazu neigen, eine instrumentelle Haltung ihren Mitbürgern und ihren politischen Tätigkeiten gegenüber einzunehmen. Eine solche Haltung gegenüber dem, was man tut, mag mit der Freiheit vereinbar sein (indem man noch freiwillig handelt), aber wenn diese Haltung einen großen Teil der eigenen Handlungen ausmacht, ergibt sich eine Art von Entfremdung sowohl anderen als auch der eigenen Tätigkeit gegenüber. Nicht, dass es nie angemessen ist, sich zu anderen im Modus des berechnenden Egoismus zu verhalten. Vielmehr gibt es Typen von sozialer Tätigkeit, bei denen eine durch Geld vermittelte Zusammenarbeit mit den spezifischen Arten von Verbindungen unvereinbar ist, die eben die Pointe solcher Tätigkeiten ausmachen. Hegel hat diesen Punkt deutlicher ausgearbeitet als Rousseau, indem er die vernünftige Gesellschaft in drei quasiautonome Sphären aufgeteilt hat, die von verschiedenen gemeinsamen Zwecken, verschiedenen Arten von Verbindungen und verschiedenen Weisen, soziale Mitarbeit zu organisieren, charakterisiert sind. Seiner Behauptung nach ist es angebracht, in der Sphäre der Wirtschaft Beziehungen nach Tauschprinzipien zu gestalten, während die gleiche Gestaltung von Beziehungen unter Familienmitgliedern und Staatsbürgern mit den kollektiveren Gesinnungen unvereinbar ist, die in diesen Sphären herrschen müssen, wenn der spezifische Zweck solcher Tätigkeiten verwirklicht werden soll. Diese Überlegung legt noch eine andere Auffassung der sozialen Pathologie nahe: Eine Gesellschaft ist krank, wenn ein Organisationsprinzip, das einer ihrer Sphären angemessen ist, gleichsam aus dieser Sphäre ausbricht und in andere Sphären „kolonisierend“ (Habermas 2011) und auf schädliche Weise eindringt. 
Dass Rousseau von der Entehrung der Legionen durch die Anstellung von Söldnern spricht, bringt einen dritten Aspekt der Pathologien Roms ans Licht, den im Anschluss an Axel Honneth eine Pathologie der misslungenen Anerkennung genannt werden könnte (Honneth 2007). Der Kern dieser Diagnose liegt in der Idee, dass es zu den Hauptaufgaben einer gesunden Gesellschaft gehört, ihren Mitgliedern eine ausreichende Vielfalt von befriedigenden Formen der Anerkennung zu bieten. Die volle Bedeutung von Rousseaus Bemerkung, die Legionen würden entehrt, wenn das Geld die Ziviltreue als Grund, dem Vaterland zu dienen, ersetzte, muss so verstanden werden: dieser erste Umstand, selbst eine Art misslungener Anerkennung, hatte die weitere Wirkung, dass auch andere Anerkennungsverhältnisse in der Gesellschaft unterhöhlt wurden, bis zu dem Punkt, wo man von einem sich selbst tragenden System von misslungener Anerkennung sprechen könnte. Besonders hervorzuheben ist die Wirkung, die die Anstellung von Söldnern auf deren eigene Selbstverständnisse hatte (und deshalb auf ihre Verständnisse dessen, was ihr eigenen Wert ausmacht): Die Söldner maßen ihren eigenen Wert nur nach dem „Preis, zu dem sie sich verkauft hatten.“ Dieser Punkt ist Mitgliedern von Gesellschaften, in denen der Markt eine herrschende Rolle im gesellschaftlichen Leben spielt, wohl bekannt: Wenn Geld zum Prinzip wird, um das herum soziale Verhältnisse organisiert werden, sind Individuen einer kaum zu widerstehenden Versuchung ausgesetzt, ihren eigenen Wert nach dem Maßstab des Geldwerts zu messen, den der Markt ihnen und ihren Dienstleistungen beimisst. Eins der Probleme damit besteht darin, dass Preise, die von Angebot und Nachfrage bestimmt sind, ethisch willkürlich sind. Genau wie der Markt auf effektive Nachfrage statt auf Bedürfnisse reagiert - er organisiert die Produktion nicht nach dem, was Menschen bedürfen, um ein gutes Leben zu führen, sondern nach den Wünschen derer, die die meisten Ressourcen haben - so ist es zufällig, ob das vom Markt verwendete Maß des persönlichen Wertes die Eigenschaften berücksichtigt, die gute Gesellschaften und gute Menschen ausmachen.

Eng verwandt mit diesem Punkt ist die Tatsache, dass Märkte den Wert von Sachen nur nach einem eindimensionalen, quantitativen Maßstab messen, nämlich, nach Preisen, die in Geldsummen ausgedrückt werden. Als Folge davon neigen Individuen dazu, sich selbst quantitativ statt qualitativ wertzuschätzen - das heißt, weniger nach den intrinsischen Verdiensten ihrer Eigenschaften und Leistungen wertzuschätzen als nach dem zufälligen Kriterium, ob sie gerade von denen nachgefragt werden, die über die zu ihrem Kauf benötigten Geldressourcen verfügen. Auch dies könnte als Entfremdung demgegenüber, wer man ist und was man tut, beschrieben werden: Man schätzt seine gesellschaftlich zuträglichen Eigenschaften nicht aufgrund einer Überzeugung, dass sie zum menschlichen Wohl beitragen, sondern weil sie Mittel sind, um ihr eigentliches Ziel zu erreichen, nämlich ihren Marktwert zu erhöhen. Hinzu kommt noch, dass, je mehr man seinen Wert nach einem quantitativen Maßstab bemisst, desto schwieriger wird es, den Wunsch, einen Wert in den Augen anderer zu haben, zu befriedigen. Im Gegensatz zu einem qualitativ bestimmten Ideal setzen uns quantitative Maßstäbe keinen natürlichen Endpunkt bei der Suche nach Bestätigung unseres Wertes. Im Prinzip lassen sich Geldpreise immer verbessern, und wenn dieser Maßstab unseres Wertes einmal verinnerlicht wird, wird Befriedigung dünn und instabil: Es gibt keinen 
Grund, nicht nach immer höheren Anerkennungssummen zu streben und daher auch keine Grenzen für das zu setzen, was Hegel eine Dynamik der „schlechten Unendlichkeit" bezeichnen würde.

Wie Rousseaus Beispiel ebenfalls nahelegt, übersetzt sich eine verzerrte Vorstellung von dem Grund des eigenen Wertes in ein entsprechend verzerrtes Bild davon, wo der Wert anderer liegt; eine falsche Selbstbewertung geht mit der misslungenen Anerkennung anderer einher. Rousseau formuliert diesen Punkt so: Sobald die angestellten Sicherheitskräfte ihren eigenen Wert nach dem Preis zu messen anfingen, zu dem sie sich verkauft hatten, verloren sie den Respekt vor dem Staat und seinen Gesetzen und auch vor ihren Mitbürgern. Das anschaulichste Zeichen dafür ist, dass der Verlust dieses Respekts es den Söldnern erlaubte, „den gezückten Dolch über ihre Mitbürger" zu halten. Aber dieser Fall von misslungener Anerkennung ist nur die Folge eines noch tiefer liegenden Falls, nämlich, dass die Magistraten aufhörten, für das Glück ihrer Subjekte zu sorgen und, weil sie „lieber Söldner kommandieren wollten als freie Menschen“, ihre Subjekte nicht mehr als Bürger anerkannten, deren grundlegendes Recht darin besteht, frei von der Willkür anderer zu sein.

Der doppelte Charakter der misslungenen Anerkennung auf Seiten der Magistraten - dass sie sowohl das Glück als auch die Freiheit ihrer Untertanen missachteten - deutet auf eine vierte Auffassung der sozialen Pathologie: die Unfähigkeit von Gesellschaftsmitgliedern, ihre beiden grundlegenden Bestrebungen - als natürliche Wesen und als frei handelnde Menschen - gleichzeitig zu befriedigen. In seiner Beschreibung der grundlegenden Aufgabe der politischen Philosophie „Finde eine Form des Zusammenschlusses, die [...] die Person und das Vermögen jedes Mitglieds verteidigt und [in der] [...] jeder [...] genauso frei bleibt wie zuvor“ (Rousseau 2010, S. 17; Rousseau 1964, S. 360) - postuliert Rousseau zwei Arten von Interessen, die ein legitimer Staat für seine Bürger befriedigen muss: ein Interesse an den Grundbedingungen des eigenen Wohls und ein Interesse an der eigenen Freiheit. Im antiken Rom, wie es hier beschrieben wird, sind Bürger zugleich unfrei und unglücklich (hungrig und überbelastet), und das ist ein Übel, sogar eine Ungerechtigkeit. Aber das ist nicht die einzige Weise, auf die eine Gesellschaft den Bestrebungen ihrer Mitglieder danach, gleichzeitig ihre Bedürfnisse befriedigt zu finden und frei zu sein, zuwiderlaufen kann. Es ist möglich, sich Institutionen vorzustellen, die ihre Mitglieder eine Wahl zwischen diesen beiden Bestrebungen aufzwingt, wo die Erreichung der einen nur auf Kosten der anderen zu haben ist. Das war die Situation der Bürger des ehemaligen Ostblocks, aber es ist auch eine passende Beschreibung der Bedingungen unter welchen U.S.-Bürgern heute eine Krankenversicherung zur Verfügung gestellt wird, die, um ihr Bedürfnis nach medizinischer Versorgung zu befriedigen, oft dem Zwang unterliegen, eine Arbeitstelle annehmen zu müssen, die ihnen übermäßig belastende Arbeitsbedingungen auferlegen, die sie in anderen Umständen schlicht ablehnen würden.

Diese Bemerkung über die medizinische Versorgung legt die Vorstellung nahe, man könne Rousseaus Behauptungen über die Zwecke des legitimen Staats auf gesellschaftliche Institutionen im Allgemeinen erweitern. Wenn man das tut, ergibt sich eine Auffassung einer gesunden Gesellschaft als einer, die unsere auf Befriedung von materiellen Bedürfnissen gerichteten Praktiken so organisiert, dass 
jene Praktiken zugleich Ausdruck unserer Freiheit sind. Im Gegensatz dazu gelten Institutionen als ,krank', die diese Einheit von Freiheit und materieller Befriedigung unmöglich machen - wie Arbeitsbedingungen, die das Arbeiten für lebensnotwendige Dinge zu einem Raum von Herrschaftsbeziehungen und der Selbstverleugnung statt zu einem Ausdruck der Freiheit machen. Dieser Ansicht nach gibt es eine soziale Pathologie immer dann, wenn die Grundbedingungen einer Gesellschaft es ihren Mitgliedern es unmöglich machen, in ihren Selbstverständnissen, in ihren Anerkennungsverhältnissen, und in ihren materiellen Praktiken ihre Mitgliedschaft im Reich der Freiheit mit der im Reich der Notwendigkeit zu vereinigen.

Das letzte gesellschaftliche Übel, das im Zusammenhang Roms zu erwähnen ist, ist jenes, das vielleicht am passendsten in der Sprache der Pathologie zu beschreiben ist. Es besteht in einer sich selbst tragenden Dynamik, die einen schlechten Zustand noch schlechter macht, und die, einmal in Gang gekommen, fast unmöglich aufzuhalten ist. Man kann eine solche Dynamik nicht anschaulicher beschreiben als Rousseau es getan hat: „Um [...] Truppen aufzustellen, musste man sie den Ackerbauern entreißen. Das Fehlen dieser Leute verminderte die Qualität der Nahrungsmittel. Deren Einfuhr führte zu Steuern, die die Preise erhöhten. Diese ersten Unordnungen ließen die Völker murren. Um sie zum Schwiegen zu bringen, musste man die Truppen verstärken und folglich das Elend. In dem Maß, wie die Hoffnungslosigkeit größer wurde, musste man sie wieder vergrößern.“

In diesem Szenario mag auch Ungerechtigkeit vorhanden sein, aber sie ist nicht das einzige Problem, das hier geschildert wird, and sie erschöpft auf keinen Fall unseren Eindruck, eine Gesellschaft, die so eine Dynamik aufweist, sei krank. Die Eigenschaft von Gesellschaften, die diese Art von Kranksein möglich macht, ist die Vorhandensein von gesellschaftlichen Kräften, die der Kontrolle der von ihnen ausgelieferten Individuen entgleiten, und die Wirkungen haben, die von den Gesellschaftsmitgliedern selbst, auch den mächtigsten unter ihnen, unbewusst erzeugt werden. Pathologien dieser Art können vorhanden sein, auch wenn niemand die schädlichen Folgen solcher gesellschaftlichen Kräfte beabsichtigt oder befürwortet. Ein freier Markt kann zum Beispiel große Ungleichheit, verbreitete Armut, und den Verlust von sozialem Zusammenhalt systematisch produzieren (und reproduzieren), ohne dass auch nur einer seiner Teilnehmer die Folgen ihrer gemeinsamen Tätigkeit voraussieht. Mehr noch: unter leicht vorstellbaren Zuständen können die zu Zeitpunkt $Z_{1}$ erzeugte Ungleichheit, Armut, und soziale Auflösung so gut wie sicher dazu führen, dass diese anfänglichen Übel zu Zeitpunkt $Z_{2}$ nur noch schlimmer sein werden. Aber, wie Rousseaus Beispiel zeigt, sind Vorgänge des Marktes nicht die einzigen gesellschaftlichen Phänomene, die eine sich selbst tragende Dynamik darstellen können, die der Bezeichnung , $\mathrm{krank}^{`}$ würdig ist.

\section{Rousseaus Kritik der modernen, ,fieberhaften' Gesellschaft}

In diesem letzten Abschnitt soll eine einzige Form von sozialer Pathologie näher untersucht werden, die in Rousseaus eigener Theorie der modernen Gesellschaft eine herausragende Rolle spielt. Diese Form von gesellschaftlicher Krankheit geht auf Platons Darstellung der fieberhaften Gesellschaft zurück, insofern die von Pla- 
ton geschilderte gesellschaftliche Krankheit ihre Quelle in gewissen erworbenen Bedürfnissen und Wünschen hat, die ohne eine gerechte politische Ordnung eine fieberhafte Gesellschaft unvermeidlich machen. Wie auch Rousseau findet Platon die Ursachen von ,sozialem Fieber' in Wünschen, die über grundlegende Bedürfnisse hinausgehen und zu unbegrenzten Wünschen nach Komfort, Luxus, Erwerb erweitert werden (Platon 2004, 372d-373e). Das Fieber der Gesellschaft, so könnte man sagen, ernährt sich von den fieberhaften Wünschen ihrer Mitglieder. Das Gleiche könnte man über die Form von gesellschaftlicher Krankheit bei Rousseau sagen, die ich jetzt untersuchen möchte, wobei er mehr über die sozialen Ursachen unbegrenzter Wünsche zu sagen hat als Platon.

Obwohl Rousseau nie von einer fieberhaften Gesellschaft redet, nennt er bestimmte Wünsche ,fieberhaft‘ (Rousseau 1997, S. 439; Rousseau 1969, S. 489490), und die Gesellschaften, deren Übel er im Zweiten Diskurs diagnostiziert, sind die, in welchen fieberhafte Wünsche herrschen. Diesem Hinweis zufolge könnte man Gesellschaften ,fieberhaft' oder krank nennen, in welchen fieberhafte Wünsche herrschen, und wo diese Wünsche gesellschaftliche Ursachen haben. Fieberhafte Wünsche sind vermutlich Wünsche, die den Organismus, dem sie eigen sind, mit schwerwiegenden Schäden, vielleicht auch mit dem Tod, bedrohen. Sie sind Wünsche, die Rousseau ,rasend', ,ungezügelt', oder ,gewaltig' nennt - Wörter, die er bei der Beschreibung der ungesunden Gesellschaft im Zweiten Diskurs großzügig verwendet. Rousseaus Schilderung von Rom enthält Beispiele von Individuen, die bösartige Wünsche haben - die Magistraten versuchen die Bürger zu unterjochen - aber, obwohl diese Wünsche für die beschriebenen Pathologien nicht irrelevant sind, sind sie nicht gerade fieberhafte Wünsche. Mit letzterem meint Rousseau Wünsche, die ungesunde Mengen von Hitze und Aufregung hervorbringen - sie sind ,rasend" - und, weil sie unbegrenzt sind, dazu neigen, sich unkontrolliert zu vergrößern, und daher als, ungezügelt' gelten. Einer Hauptthese des Zweiten Diskurses zufolge sind fieberhafte Wünsche von einer einzigen menschlichen Leidenschaft verursacht, von der amour-propre - oder, etwas genauer, von den ungesunden Formen dieser Leidenschaft, die er ,entfacht ' nennt. ${ }^{5}$

$\mathrm{Da}$ ich die amour-propre und ihre entfachten Formen an anderer Stelle ausführlich behandelt habe (Neuhouser 2012), beschränke ich mich hier auf die Aspekte seiner Position, die einem Verständnis der bestimmten sozialen Pathologie, die aus den fieberhaften Wünschen der Gesellschafsmitglieder entstehen, wesentlich sind. Besonders relevant ist folgende Textstelle, in der Rousseau seine Auffassung der fieberhaften Gesellschaft darstellt:

„Alle unsere Fähigkeiten wären nun entwickelt, [...] die amour propre interessiert, die Vernunft aktiviert und der Geist beinahe an der Grenze der Vollkommenheit angelangt, deren er fähig ist. [...] [D]er Rang [...] eines jeden Menschen [wäre] festgelegt, nicht nur in Bezug auf die Menge der Güter und die Macht [...] sondern auch in Bezug auf den Geist, die Schönheit, die Stärke oder die Gewandtheit [...]; und da

5 Den Ausdruck, enflamer ' [hier wiedergegeben mit, entfacht'] verwendet Rousseau nur ein einziges Mal, um l'amour propre einzuschränken (Rousseau 1997, S. 510; Rousseau 1969, S. 540). Diese Terminologie ist vor allem Dent geschuldet, der den Ausdruck häufig verwendet und dazu neigt, ,entfacht' mit ,exzessiv' und ,pervertiert' gleichzusetzen (Dent 1989, S. 52, 256). 
diese Eigenschaften die einzigen waren, die einem Achtung verschaffen konnten, war es bald notwendig, sie zu haben oder sie vorzutäuschen [...]. [Der Mensch], der früher [...] unabhängig war, jetzt durch eine Vielzahl neuer Bedürfnissen [...] seinen Mitmenschen [Untertan] [...]. Er muss [...] unablässig danach trachten, [andere] für sein Schicksal zu interessieren und sie ihren Profit [...] darin finden zu lassen, dass sie für den seinen arbeitet: Das macht ihn betrügerisch und hinterlistig gegen die einen, herrisch und hart gegen die anderen. [...] [D]er Eifer, sein relatives Vermögen zu erhöhen - weniger aus einem wahrhaften Bedürfnis heraus, als um sich über die anderen zu setzen -, [gibt] allen Menschen einen finsteren Hang ein, sich wechselseitig zu schaden. [...] Konkurrenz und Rivalität, [...] das versteckte Verlangen seinen Profit auf Kosten anderer zu machen; alle diese Übel [...] sind das untrennbare Gefolge der entstehenden Ungleichheit“ (Rousseau 1993, S. 207, 209; Rousseau 1964, S. 174 $175)$.

Diese Textstelle schreibt der kranken Gesellschaft eine große Anzahl von Übeln zu, von denen viele uns aus dem Beispiel Roms schon bekannt sind, nämlich: Unterjochung; intensiver Wettkampf; eine Gesellschaft, in der jede Verbindung auf selbstinteressierter Berechnung gegründet ist; der beständige Wunsch, das eigene Los zu verbessern, um sich über andere zu stellen; und verschiedene Formen von Lastern: Betrug, Heuchelei, und der Wille, anderen Schäden zuzufügen. Wie bereits oben erwähnt worden ist, findet Rousseau die Ursache dieser Übel in den fieberhaften Gestaltungen einer einzigen Leidenschaft, der amour-propre.

Diese These kann erläutert werden, indem man sich auf einen Aspekt der hier beschriebenen Übel konzentriert, nämlich den Wunsch der Gesellschaftsmitglieder, ihr eigenes Los zu verbessern, um sich über andere zu stellen - ein Wunsch, der die perverse Folge hat, dass Individuen „die Dinge, die sie genießen, nur soweit schätzen, als die anderen sie entbehren, und die [...] aufhören würden glücklich zu sein, wenn [die Anderen] aufhörte[n], elend zu sein“ (Rousseau 1993, S. 257; Rousseau 1964, S. 189). Wenn die oben aufgestellten Behauptungen richtig sind, sollte es möglich sein, zu erklären: warum dieser Zustand von einer fieberhaften Gestaltung der amour-propre abhängt; wie solche fieberhaften Bestrebungen die beschriebenen Übel hervorbringen; und in welchem Sinne diese Übel soziale Pathologien ausmachen.

In diesem Zusammenhang ist die wichtigste Eigenschaft der amour-propre ihre ,relative' Natur. Die amour-propre ist relativ - relativ zu anderen Subjekten - in zwei Hinsichten. Erstens ist das von der amour propre angestrebte Gut wesentlich vergleichend: Anerkennung zu begehren bedeutet, eine bestimmte Stellung im Vergleich zu der Stellung anderer zu begehren. ${ }^{6}$ Das Ziel der amour-propre ist also ein stellungsrelatives Gut, und das impliziert: Der eigene Erfolg (anerkannt zu werden) besteht in einem Erfolg im Vergleich zu anderen. Die zweite Hinsicht, in der die amour-propre relativ ist, besteht darin, dass zu ihrer Befriedigung die Meinungen jener Mitmenschen erforderlich sind, deren Anerkennung man anstrebt. Beide dieser relativen Eigenschaften der amour-propre tragen zu ihrer Macht bei,

6 „[S]obald sich l'amour propre entwickelt hat, das relative Ich unabläßlich ins Spiel tritt, und dass der Jüngling die anderen nie anders als in Bezug auf sich selbst beobachtet und sich mit ihnen vergleicht“ (Rousseau 1997, S. 502; Rousseau 1969, S. 534). 
,die schlimmsten Dinge unter den Menschen hervorzubringen, aber für die soziale Pathologie, die ich hier untersuchen möchte, ist die erste - ihre vergleichende Natur - die wichtigere.

Ehe diese These erläutert werden kann, muss noch ein Punkt besprochen werden, der sowohl die Wichtigkeit des Ziels der amour-propre erklärt, als auch den Grund, warum die von ihr abgeleiteten Wünsche für die Menschen, denen sie eigen sind, als Bedürfnisse auftreten. Dabei ist es wichtig, dass Rousseaus Auffassung der elementaren Arten von Antrieben, die Menschen auszeichnen, der amour-propre denselben grundlegenden Status zuschreibt, den er auch dem Trieb nach Selbsterhaltung zukommen lässt. Diese Gleichwertigkeit der beiden Grundtriebe ist dadurch zu erklären, dass es in beiden Formen der Selbstliebe um das Sein des Selbst geht. Bei dem Trieb nach Selbsterhaltung ist dies selbstverständlich, weil physisches Überleben sein höchstes Ziel ist, aber es gilt nicht weniger auch für die amour-propre, die auf das ,moralische' Überleben des Selbst abzielt.

Es ist diese Idee, die hinter Rousseaus Bemerkung steckt, man gewinne durch die Anerkennung ein „Gefühl der eigenen Existenz" (Rousseau 1993, S. 271; Rousseau 1964, S. 193. Hervorhebung F. N.). Nicht anerkannt zu werden, stellt freilich keine Gefahr für die eigene physische Existenz - und doch ist eine Person, die in den Augen anderer keine Stellung hat - wie sich auch in unserer Alltagssprache zeigt - ein ,Niemand'. Die hier fragliche Art von Existenz ist das, was Sartre das „Für-Andere-Sein“ nennt, und Anzeichen eines solchen Seins ist nicht die physische sondern die ,moralische Präsenz ${ }^{6}{ }^{7}$ wie sie sich in dem Einfluss äußert, den man (als ein geschätztes Subjekt) auf das Verhalten anderer hat. Wenn, allgemein gesprochen, das Vermögen, Wirkungen hervorzurufen, ein Anzeichen der Wirklichkeit eines Wesens ist, dann bedeutet, von anderen wertgeschätzt zu sein - insofern sich dies in deren Äußerungen und Handlungen ausdrückt - einem Selbst Wirklichkeit einer nicht-physischen, dezidiert menschlichen Art zu verleihen.

Diese Eigenschaft der amour-propre erklärt sowohl die Grimmigkeit, mit der sie Menschen anregen kann, als auch ihre Fähigkeit, alle Lebensbereiche zu infizieren und alltägliche Tätigkeiten in Bestrebungen nach Anerkennung zu verwandeln. Fast alles, was man tut - essen, sich kleiden, Eigentum erwerben - kann dazu dienen, Eigenschaften des Selbst zur Schau zu tragen, die die eigene Stellung in den Augen der anderen verbessern. Um aber die sozialen Pathologien zu erklären, die hier von Interesse sind - der maßlose Wettkampf; endlose Bemühungen, andere zu übertreffen; übelwollender Konflikt; und die Unfähigkeit, Befriedigung zu finden - muss diese Eigenschaft der amour-propre in Zusammenhang mit ihrer vergleichenden Natur gebracht werden. Es ist diese Beschaffenheit der amourpropre, in der Rousseau die Antwort auf die Hauptfrage des zweiten Diskurses erblickt: „Wo kommt die soziale Ungleichheit her? “ Seine Strategie, diese Frage zu beantworten, besteht darin, den Bestandteil der menschlichen Psychologie zu finden, die erklärt, warum wir die Ungleichheit anstreben. Es ist leicht einzusehen, warum die amour-propre für diese Aufgabe gut geeignet ist: Da die von ihr angestrebte Stellung immer in Bezug auf die Stellung anderer bestimmt wird, kann sie

7 Der Ausdruck stammt von Dent, der auf hervorragende Art und Weise erläutert, worin eine solche Präsenz besteht (Dent 1989, S. 21-26, 49-50, 56-58). 
uns mit einem Anreiz ausstatten, nicht nur selbst gut abzuschneiden sondern besser als andere abzuschneiden. Das erklärt, wie es möglich ist, dass soziale Ungleichheit unser Werk (statt das der Natur) ist: Sobald wir meinen, eine Bestätigung unseres eigenen Werts erfordere eine Anerkennung unserer Überlegenheit über andere, ist Ungleichheit nötig, um die amour-propre zu befriedigen.

Dann ist das pathologische Potenzial der amour-propre leicht zu verstehen: Wenn der Wunsch nach Anerkennung so gestaltet ist, dass er uns dazu antreibt, nach einer überlegenen Stellung zu suchen, sind Frust, Konflikt, Konkurrenz, und Laster geradezu garantiert. ${ }^{8}$ Denn wenn Erfolg dadurch definiert wird, dass man als überlegen anerkannt wird, wird eine allgemeine Befriedigung der amour-propre unmöglich, weil Anerkennung zu einem knappen Gut wird, wenn mehrere eine überlegene Stellung anstreben. Wenn einige eine überlegene Stellung erreichen sollen, dann müssen sich andere mit einer unterlegenen Stellung begnügen. Anstatt für alle verfügbar zu sein, wird Anerkennung zu einem Gegenstand endloser Konkurrenz und enttäuschter Verlangen.

Ein zweites Problem ist das des ,Rattenrennens` oder des Konkurrenzkampfes. Dieses Problem ergibt sich daraus, dass selbst wenn Überlegenheit erlangt wird, sie unsicher und kurzlebig ist, solange sie im Vergleich zu anderen erreicht wird, die dasselbe begehren. Um den Konkurrenten zu übertreffen, der mich gerade überholt hat oder die Vorrangstellung zu behaupten, die ich momentan einnehme, muss ich ständig darauf aus sein, meine gegenwärtige Stellung zu verbessern. In einer solchen Situation lastet auf den Individuen ein nahezu grenzenloser Druck, ihre Position zu verbessern und damit die Fortschritte ihrer Rivalen auszugleichen oder vorwegzunehmen, was zu einem rastlosen und unaufhörlichen Sich-Übertrumpfen führt. Das Problem damit ist nicht bloß, dass die einzige Befriedigung, die die amour-propre findet, flüchtig bleiben muss, sondern auch dass Begierden auf eine Art und Weise grenzenlos werden, die dem menschlichen Glück zuwiderläuft. Solche sich stets ausweitende Begierden zwingen diejenigen, denen sie eigen sind, das Erfordernis auf, große Teile ihrer Energie dafür aufzuwenden, die Güter und Ehren zu verfolgen, von denen sie hoffen, sie könnten ihr Streben nach Überlegenheit befriedigen. Doch wie ausgearbeitet solche Vorhaben auch sein mögen, sind sie zum Scheitern verurteilt, erstens, weil die für sie erforderliche Arbeit in der Regel die Befriedigung überwiegt, die sie mit sich bringen, und zweitens weil die Individuen, sobald ihre Motivationen in diesem Ausmaß von fieberhafter amour-propre durchdrungen sind, die Fähigkeit verlieren, ihre Errungenschaften wegen der intrinsischen (nicht-relativen) Freuden zu genießen, die sie gewähren.

Schließlich neigt das Streben nach einer überlegenen Stellung dazu, Laster hervorzurufen, vor allem die Neigung, anderen zu schaden oder an ihrem Unglück Vergnügung zu finden: Messe ich meine Stellung im Vergleich zu der von anderen, kann ich meine eigene Stellung voranbringen, entweder indem ich mein Los verbessere oder indem ich das der anderen verschlechtere. Fieberhafte amour-propre stattet uns also mit einem Anreiz aus, das Unglück anderer anzustreben. Es gibt ein zweites Laster, das die amour-propre verursachen kann: den Willen andere zu

8 Eigentlich ist Rousseaus Auffassung komplizierter, denn er betrachtet einige Formen des Wunsches nach überlegener Stellung als harmlos. 
beherrschen. Anstatt den Willen, andere zu beherrschen, als einen grundlegenden Drang der menschlichen Natur zu betrachten, erklärt Rousseau ihn als eine Entstellung der amour-propre, die zwar eine verbreitete, keineswegs aber eine notwendige menschliche Eigenschaft ist. Das wird ihm dadurch möglich, dass er den Willen, andere zu beherrschen, als im Grunde ein Verlangen nach Anerkennung einer Art von überlegener Stellung begreift: Sich selbst zum Herrn der Willen anderer zu machen, ist eine Art, öffentliche Bestätigung des eigenen bevorzugten Stellenwerts zu finden - nicht nur im Verhalten der Gehorchenden, sondern auch in den Augen derer, die Zeugen dieses Gehorsams sind.

Es bleibt zu erklären, warum diese fieberhaften Gestaltungen der amour-propre und ihre Folgen, soziale statt bloß individuelle Pathologien darstellen. Es gibt eine offensichtliche Hinsicht, in der jede Erscheinungsform der amour-propre ein soziales Phänomen ist. Das folgt aus ihrer relativen Natur. Jedes aus der amour-prop$r e$ herkommende Verlangen bezieht sich notwendigerweise auf andere in zwei Hinsichten: Anerkennung kommt von anderen und wird in Bezug auf andere angestrebt. Doch diese Tatsache reicht nicht aus, alle aus der amour-propre entstehenden Pathologien als soziale Pathologien gelten zu lassen. Um als eine soziale Pathologie zu gelten, muss die fragliche Krankheit, entweder gesellschaftlichen Verhältnissen innewohnen oder durch sie verursacht sein (oder beides). Diese beiden Ideen - dass eine Krankheit gesellschaftlichen Verhältnissen innewohnen, und dass sie eine gesellschaftliche Ursache haben kann - sollen deutlicher werden, wenn erklärt wird, warum es sinnvoll ist, die von der amour-propre hervorgebrachten Übel als soziale Pathologien zu betrachten.

Es ist plausibel, das Verlangen nach einer überlegenen Stellung als eine Pathologie zu verstehen, soweit es einen dazu bringt, ,die Dinge, die man genießt, nur soweit zu schätzen, als die anderen sie entbehren“" (Rousseau 1993, S. 257; Rousseau 1964, S. 189). Aber die meisten Übel, die aus einem solchen Verlangen hervorkommen - übelwollender Wettbewerb, Konflikt, und Frust - erfordern eine Vielzahl von Subjekten, deren Begierden nach Anerkennung ähnlich gestaltet sind. Die Konkurrenz, das ,Rattenrennen', der Kriegszustand - alle sind soziale Phänomene, weil sie in Verhältnissen unter Menschen bestehen und von einer gewissen Dynamik unter ihnen abhängen. Sie sind Übel, die gesellschaftlichen Verhältnissen statt den Seelen oder Handlungen von Individuen innewohnen.

Doch dies allein erschöpft den Sinn nicht, in dem die fraglichen Pathologien sozial sind, und zwar deshalb, weil die fieberhaften Begierden, die sie hervorbringen, eine gesellschaftliche Ursache haben. Rousseau unterscheidet zwischen zwei möglichen Quellen von fieberhaften Begierden nach Anerkennung: gesellschaftlichen Institutionen (im Zweiten Diskurs) und häuslicher Erziehung (im Emile). Da die mit der amour-propre verbundenen Probleme zugleich gesellschaftliche und häusliche Ursachen haben, sind sie allein durch gesellschaftliche Reformen nicht zu beseitigen. (Und das erklärt den größten Teil von Rousseaus pessimistischem Glauben bezüglich der Möglichkeit, die von ihm diagnostizierten Übel definitiv zu beheben.) Da es hier um die soziale Pathologie geht, werde ich im Folgenden nur die gesellschaftlichen Ursachen der fieberhaften amour-propre berücksichtigen.

In diesem Zusammenhang gilt es wieder an die eben zitierte Textstelle zu denken, in der Rousseau, gleich nachdem er die Ursprünge der Arbeitsteilung, der 
verbesserten Produktivkräfte, und des Privateigentums darstellt, auch die fieberhafte Gesellschaft beschreibt:

\begin{abstract}
„Alle unsere Fähigkeiten wären nun entwickelt, [...] die amour propre interessiert. [...] [D]er Rang [...] eines jeden Menschen [wäre] festgelegt, nicht nur in Bezug auf die Menge der Güter und die Macht [...] sondern auch in Bezug auf den Geist, die Schönheit, die Stärke oder die Gewandtheit [...]; und da diese Eigenschaften die einzigen waren, die einem Achtung verschaffen konnten, war es bald notwendig, sie zu haben oder sie vorzutäuschen [...]“ (Rousseau 1993, S. 207; Rousseau 1964, S. 174. Hervorhebung F. N.).
\end{abstract}

Der Schlüssel dafür, die gesellschaftlichen Ursachen der fieberhaften amour-propre zu verstehen, liegt in dem Satz „da diese Eigenschaften die einzigen waren, die einem Achtung verschaffen konnten “. Der Punkt, der von diesem Satz nahegelegt wird, ist, dass die amour-propre - einschließlich einiger Formen des Verlangens, als überlegen anerkannt zu werden - die hier beschriebenen Übel nur dann hervorbringt, wenn ein gewisser Hintergrundszustand gegeben ist, nämlich das Fehlen von Möglichkeiten, Formen von Anerkennung zu erlangen, die gütiger sind als die, die den Individuen in der fieberhaften Gesellschaft des Zweiten Diskurses zur Verfügung stehen. Mit anderen Worten hat die Natur einer bestimmten Gesellschaft Auswirkungen auf die Folgen, die die amour-propre in ihr haben wird, und zwar dadurch, dass sie das Feld der Möglichkeiten gestaltet, innerhalb dessen Anerkennung angestrebt werden kann. Das Problem mit der im Zweiten Diskurs beschriebenen Gesellschaft liegt darin, dass sie ihren Mitgliedern keine Praktiken bietet, innerhalb derer sie eine Stellung für andere erwerben können, die es nicht erforderlich machen, besser als ihre Mitbürger abschneiden zu wollen. Außerdem sind es die schädlichsten Formen von Überlegenheit, wie Opulenz und Herrschaft, die in einer solchen Gesellschaft das meiste Ansehen mit sich bringen. Noch einmal: Wie Individuen versuchen, ihre amour-propre zu befriedigen, hängt von den Anerkennungsmöglichkeiten ab, die ihre Gesellschaft ihnen zur Verfügung stellt. Eine Gesellschaft, die Reichtumsunterschiede in Grenzen hält, bringt in Individuen andere Anerkennungswünsche hervor, als eine, deren Wirtschaftssystem sich von dem Wunsch ihrer Mitglieder ernährt, stinkreich` zu werden; eine Gesellschaftsordnung, die auf geerbten Ständeprivilegien beruht, regt Individuen an, das ,Gefühl der eigenen Existenz' anders zu suchen, als eine, die jede Person als gleichberechtigt anerkennt. Mit anderen Worten haben gesellschaftliche Institutionen bildende Wirkungen auf ihre Mitglieder, und das erklärt, warum die von der amour-propre hervorgebrachten Übel als sozial verursacht und daher als soziale Pathologien gelten.

Rousseaus Behauptung, die fieberhafte amour-propre habe gesellschaftliche Ursachen, impliziert, dass es für eine Reformierung der Gesellschaft nötig ist, Institutionen zu schaffen, die allen stabile und gütige Formen von Anerkennung zur Verfügung stellen. Das kann in der Tat als eine Hauptaufgabe des Gesellschaftsvertrags angesehen werden. Um dies zu verdeutlichen, muss man sich wieder an die vergleichende Natur der amour-propre wenden. Beim Nachdenken über die möglichen Heilmittel gegen die von der amour-propre hervorgebrachten Übel gilt es zu beachten, dass eine relative Stellung nicht notwendigerweise eine überlegene 
ist. Wenn uns die amour propre einfach dazu bringt, die Achtung zu begehren, die wir als Menschen verdienen - Achtung, die man im Gegenzug anderen zu gewähren bereit ist -, dann ist diese begehrte Stellung zwar relativ, aber keine überlegene Stellung. Eine gleiche Stellung ist immerhin eine vergleichende Stellung (Cohen 1997, S. 108-111). Das heißt, eine wohlgeordnete (gesunde) Gesellschaft kann sehr viel dazu beitragen, das Bedürfnis ihrer Mitglieder nach Anerkennung zu befriedigen, indem sie ihre Institutionen derart gestaltet, dass allen Mitgliedern ein gleiches Maß an Achtung gewährt wird, was wiederum Auswirkungen auf die Selbstverständnisse derer hat, die in jener Gesellschaft aufwachsen - einschließlich auf die Weisen, auf die sie versuchen, ihr Bedürfnis nach Anerkennung zu befriedigen. Fieberhafte Begierden nach Anerkennung können wesentlich weniger fieberhaft gemacht werden, indem Institutionen sie auf gesunde statt auf pathologische Weise gestalten und in ihren gesunden Formen befriedigen. Es ist dann leicht zu sehen, wie der Gesellschaftsvertrag auf einige der von der amour-propre hervorgebrachten Probleme eingeht. Denn der Kern von Rousseaus Auffassung des allgemeinen Willens ist die moralische Gleichheit aller, die der legitime Staat in vier Hinsichten verwirklicht: alle Bürger sind vor dem Gesetz gleich; jeder hat dieselben negativen Freiheitsrechte wie alle andere; alle haben gleiche Rechte auf politische Teilnahme; und, am allerwichtigsten, Gesetze gelten nur dann als legitim, wenn sie die grundlegenden Interessen aller Bürger schützen.

Zum Schluss sei noch erwähnt, dass diese liberalen, politischen Maßnahmen Rousseaus seine Antwort auf die von der fieberhaften amour-propre hervorbrachten Probleme nicht erschöpfen können. Denn aus Gründen, auf die hier nicht eingegangen werden kann, kann der moralische Respekt allein unsere Wünsche, von anderen geschätzt zu werden, nicht befriedigen. Das bedeutet, dass es auch innerhalb einer vollkommen liberalen Gesellschaft viel Raum für soziale Pathologien gibt, die gleiche politische Rechte allein nicht heilen können. In dem letzten Abschnitt dieses Aufsatzes habe ich versucht, dem Begriff der sozialen Pathologie einen Inhalt zu geben und mit Hilfe von Ideen aus dem Gesellschaftsvertrag kurz anzudeuten, wie ein gutes Gestalten gesellschaftlicher Institutionen Heilmittel gegen solche Pathologien sein könnte, vor allem indem sie die von den Gesellschaftsmitgliedern erworbenen Begierden nach Anerkennung auf gesunde Weise gestalten. Wenn Rousseau damit recht hat, dass politische Maßnahmen allein nicht alle sozialen Pathologien heilen können, die die amour-propre erzeugen kann, dann muss die liberale politische Philosophie durch eine Sozialtheorie ergänzt werden, die erklärt, wie auf ähnlich gesunde Weise nichtpolitische Institutionen, jene Verlangen nach Anerkennung gestalten können, die von moralischem Respekt allein nicht zu befriedigen sind. Hegel war der erste Philosoph, der sich dieser Aufgabe systematisch widmete, aber die Herausforderung selbst, einschließlich der Idee der sozialen Pathologie, hat er von Rousseau geerbt. 


\section{Literatur}

Cohen, Joshua. 1997. The Natural Goodness of Humanity. In Reclaiming the History of Ethics - Essays for John Rawls, Hrsg. Andrews Reath, Barbara Herman und Christine M. Korsgaard, 102-139. Cambridge: Cambridge University Press.

Dent, N. J. H. 1989. Rousseau. An Introduction to His Psychological, Social and Political Theory. Oxford: Basil Blackwell.

Habermas, Jürgen. 2011. Theorie des Kommunikativen Handelns. Frankfurt: Suhrkamp.

Honneth, Axel. 2007. Pathologien der Vernunft. Geschichte und Gegenwart der kritischen Theorie. Frankfurt a. M.: Suhrkamp.

Neuhouser, Frederick. 2012. Pathologien der Selbstliebe. Freiheit und Anerkennung bei Rousseau. Berlin: Suhrkamp.

Neuhouser, Frederick. 2009. Die normative Bedeutung von ,Natur' im moralischen und politischen Denken Rousseaus. In Sozialphilosophie und Kritik, Hrsg. Martin Hartmann, Rahel Jaeggi, Martin Saar und Rainer Forst, 109-133. Frankfurt am Main: Suhrkamp.

Platon. 2004. Der Staat. In Sämtliche Dialoge, Hrsg. Otto Apelt. Hamburg: Meiner.

Rousseau Jean-Jacques. 1964. Oeuvres Complètes, Bd. 3, Hrsg. Bernard Gagnebin und Marcel Raymond. Paris: Gallimard, Bibliothèque de la Pléiade.

Rousseau Jean-Jacques. 1969. Oeuvres Complètes, Bd. 4, Hrsg. Bernard Gagnebin und Marcel Raymond. Paris: Gallimard, Bibliothèque de la Pléiade.

Rousseau, Jean-Jacques. 1977. Politische Schriften, Bd. 1. Übers. Ludwig Schmidts. Paderborn: Ferdinand Schöningh.

Rousseau, Jean-Jacques. 1997. Emile oder von der Erziehung. Übers. Siegfried Schmitz. Düsseldorf: Artemis und Winkler.

Rousseau, Jean-Jacques. 1993. Diskurs über den Ursprung und die Grundlagen der Ungleichheit unter den Menschen. Übers. Heinrich Meier. Paderborn: Schöningh.

Rousseau, Jean-Jacques. 1995. Vom Geselligkeitszustand des Menschengeschlechts überhaupt [Auszüge aus dem Genfer Manuskript]. In Schriften zur Kulturkritik. Übers. Kurt Weigand. Hamburg: Meiner.

Rousseau, Jean-Jacques. 2010. Vom Gesellschaftsvertrag. Übers. Hans Brockard. Stuttgart: Reclam.

\section{Autorenangaben}

Frederick Neuhouser, Professor of Philosophy, Columbia University, Department of Philosophy, Barnard College, 3009 Broadway, New York, New York 10027, USA

fneuhous@barnard.edu 